\title{
OPEN SARS-CoV-2 infection hospitalization, severity, criticality, and fatality rates in Qatar
}

\author{
Shaheen Seedat $t^{1,2,3,11}$, Hiam Chemaitelly ${ }^{1,2,11}$, Houssein H. Ayoub ${ }^{4,11}$, \\ Monia Makhoul1,2,3, Ghina R. Mumtaz ${ }^{5}$, Zaina Al Kanaani ${ }^{6}$, Abdullatif Al Khal ${ }^{6}$, \\ Einas Al Kuwari ${ }^{6}$, Adeel A. Butt ${ }^{3,6}$, Peter Coyle ${ }^{6}$, Andrew Jeremijenko ${ }^{6}$, \\ Anvar Hassan Kaleeckal ${ }^{6}$, Ali Nizar Latif ${ }^{6}$, Riyazuddin Mohammad Shaik ${ }^{6}$, \\ Hadi M. Yassine ${ }^{7,8}$, Mohamed G. Al Kuwari ${ }^{9}$, Hamad Eid Al Romaihi ${ }^{10}$, \\ Mohamed H. Al-Thani ${ }^{10}$, Roberto Bertollini ${ }^{10}$ \& Laith J. Abu-Raddad ${ }^{1,2,3 凶}$
}

The SARS-CoV-2 pandemic resulted in considerable morbidity and mortality as well as severe economic and societal disruptions. Despite scientific progress, true infection severity, factoring both diagnosed and undiagnosed infections, remains poorly understood. This study aimed to estimate SARS-CoV-2 age-stratified and overall morbidity and mortality rates based on analysis of extensive epidemiological data for the pervasive epidemic in Qatar, a country where $<9 \%$ of the population are $\geq 50$ years. We show that SARS-CoV- 2 severity and fatality demonstrate a striking age dependence with low values for those aged $<50$ years, but rapidly growing rates for those $\geq 50$ years. Age dependence was particularly pronounced for infection criticality rate and infection fatality rate. With Qatar's young population, overall SARS-CoV- 2 severity and fatality were not high with $<4$ infections in every 1000 being severe or critical and $<2$ in every 10,000 being fatal. Only 13 infections in every 1000 received any hospitalization in acute-care-unit beds and $<2$ in every 1000 were hospitalized in intensive-care-unit beds. However, we show that these rates would have been much higher if Qatar's population had the demographic structure of Europe or the United States. Epidemic expansion in nations with young populations may lead to considerably lower disease burden than currently believed.

The pandemic caused by the severe acute respiratory syndrome coronavirus 2 (SARS-CoV-2) infection and associated Coronavirus Disease 2019 (COVID-19) continue to be a global health challenge ${ }^{1}$. Aside from direct implications on morbidity and mortality ${ }^{1}$, the pandemic led to severe economic and societal disruptions ${ }^{2}$, a consequence of the social and physical distancing restrictions imposed to slow SARS-CoV-2 transmission in view of its severity and fatality.

One of the most affected countries by this pandemic is Qatar, a peninsula in the Arabian Gulf region with a population of 2.8 million $^{3,4}$. With its unique demographic and residential dwelling structure where $60 \%$ of the population are expatriate craft and manual workers (CMWs) living in large shared housing accommodations ${ }^{3,4}$, Qatar experienced a pervasive epidemic with $>60,000$ laboratory-confirmed infections per million population as of November 23, 20205,6. The epidemic grew rapidly starting from March 2020, peaked in late May 2020, then rapidly declined in subsequent weeks, and had been in a stable low-incidence phase up to end of 2020

${ }^{1}$ Infectious Disease Epidemiology Group, Weill Cornell Medicine-Qatar, Cornell University, Doha, Qatar. ${ }^{2}$ World Health Organization Collaborating Centre for Disease Epidemiology Analytics On HIVIAIDS, Sexually Transmitted Infections, and Viral Hepatitis, Weill Cornell Medicine-Qatar, Cornell University, Qatar Foundation - Education City, P.O. Box 24144, Doha, Qatar. ${ }^{3}$ Department of Population Health Sciences, Weill Cornell Medicine, Cornell University, New York, NY, USA. "'Department of Mathematics, Statistics, and Physics, Qatar University, Doha, Qatar. ${ }^{5}$ Department of Epidemiology and Population Health, American University of Beirut, Beirut, Lebanon. ${ }^{6}$ Hamad Medical Corporation, Doha, Qatar. ${ }^{7}$ Biomedical Research Center, Qatar University, Doha, Qatar. ${ }^{8}$ Department of Biomedical Science, College of Health Sciences, Member of QU Health, Qatar University, Doha, Qatar. ${ }^{9}$ Primary Health Care Corporation, Doha, Qatar. ${ }^{10}$ Ministry of Public Health, Doha, Qatar. ${ }^{11}$ These authors contributed equally: Shaheen Seedat, Hiam Chemaitelly and Houssein H. Ayoub. ${ }^{\square}$ email: Ija2002@ qatar-med.cornell.edu 
(Supplementary Figure S1). A series of serological studies completed by November 23, 2020 suggested that about half of the population have already been infected ${ }^{6-9}$.

With a well-resourced public healthcare structure and a centralized and standardized data-capture system for all SARS-CoV-2 testing and COVID-19 care, Qatar has one of the most extensive databases to characterize this epidemic and its toll ${ }^{6}$. In addition to large-scale polymerase chain reaction (PCR) and serological testing, multiple population-based PCR and serological surveys have been conducted to date ${ }^{6-9}$. As of November 23 , 2020, cumulative overall testing rates exceeded 638,000 per million population for PCR and 105,000 per million population for antibodies ${ }^{10}$. A comprehensive clinical characterization has also been completed for the hospitalized COVID-19 cases through individual chart reviews by trained medical personnel, including infection severity classification as per the World Health Organization (WHO) guidelines ${ }^{11}$.

Given the pervasive and advanced nature of the epidemic and availability of extensive epidemiological data, Qatar provides a unique opportunity to assess the extent of SARS-CoV-2 morbidity and fatality. We aimed in this study to estimate the age-stratified and overall infection acute-care and intensive-care-unit (ICU) hospitalization rates, infection severity and criticality rates, and infection fatality rate.

\section{Methods}

Mathematical model and parameterization. Building on our previously developed SARS-CoV-2 models ${ }^{12-18}$, an age-structured deterministic mathematical model was constructed to describe SARS-CoV-2 transmission dynamics and disease progression in the population from the epidemic onset up to November 22, 2020 (Supplementary Figure S2). Susceptible individuals in each age group were at risk of acquiring the infection based on their infectious contact rate per day, age-specific susceptibility to the infection, and an age-mixing matrix defining mixing between individuals in the different age groups. Following a latency period, infected individuals developed an infection that either did not require hospitalization, or that required hospitalization in an acute-care bed or in an ICU bed. Individuals admitted to an ICU bed had an additional risk for COVID-19 mortality. The model further included compartments tracking infection severity (asymptomatic/moderate/mild infection, severe infection, or critical infection as per WHO severity classification) ${ }^{11}$. Population movement between model compartments was described using a set of coupled nonlinear differential equations (Supplementary Text S1).

The model was parameterized using best available data for SARS-CoV-2 natural history and epidemiology. Model parameters, definitions, and justifications can be found in Supplementary Tables S1 and S2. The size and demographic structure of the population of Qatar were based on a population census conducted by Qatar's Planning and Statistics Authority ${ }^{3}$.

Model fitting and analyses. The model was fitted to extensive time-series and age-stratified data for PCR laboratory-confirmed infections, PCR testing positivity rate, antibody testing positivity rate, PCR and serological surveys, daily hospital admissions in acute-care and ICU beds, hospital occupancy in acute-care and ICU beds, incidence of severe and critical infections as per WHO classification ${ }^{11}$, and COVID-19 deaths (further details in Supplementary Text S2). A Bayesian method, based on the incremental mixture importance sampling with shot-gun optimization ${ }^{19,20}$, was used to fit the model to the different data sources and to estimate the mean and $95 \%$ credible interval (CI) for each estimated parameter (Supplementary Text S2), such as the mean duration of acute-care hospitalization and the mean duration of ICU-care hospitalization. The model was coded, fitted, and analyzed using MATLAB R2019a $\mathrm{a}^{21}$.

Outcome measures. Table 1 provides a listing of each outcome measure estimated in this study, its definition, and its interpretation. Two sets of outcome measures were generated. The first set includes crude case rates, such as the crude case fatality rate, calculated as the cumulative number of a disease outcome (say COVID-19 death) over the cumulative number of documented (that is PCR laboratory-confirmed) infections.

The second set includes infection rates, such as the infection fatality rate, calculated as the cumulative number of a disease outcome (say COVID-19 death) over the model-estimated cumulative number of infections, documented and undocumented.

Two separate criteria for classifying morbidity were used: one based on actual recorded hospital admission (acute-care or ICU) and one based on clinical presentation as per WHO classification of disease severity. While the two are overlapping with severe cases typically admitted to acute-care beds, and critically ill cases admitted to ICU beds, a significant fraction of mild or moderately ill cases were hospitalized out of caution. Moreover, hospitalization was used as a form of case isolation earlier in the epidemic. Of note that the health system in Qatar remained well within its threshold even at the epidemic peak towards end of May 2020.

The diagnosis (detection) rate was further calculated as the cumulative number of infections that were documented (that is with PCR laboratory-confirmed diagnosis) over the model-estimated cumulative number of infections, documented and undocumented.

Ethical approvals. This study was approved by the HMC and Weill Cornell Medicine-Qatar Institutional Review Boards.

\section{Results}

Figure 1A shows the crude case acute-care and ICU bed hospitalization rate versus time from the epidemic onset up to November 22, 2020. The rate was rather stable, but with a slightly declining trend, and was assessed at 113.9 acute-care and/or ICU hospital admissions per 1000 laboratory-confirmed infections on November 22, 2020. As of this date, a total of 18,509 acute-care and 1759 ICU hospital admissions had been registered. 


\begin{tabular}{|c|c|c|}
\hline Outcome measure & Definition & Interpretation \\
\hline \multicolumn{3}{|l|}{ Crude case rates-data estimation } \\
\hline 1. Crude acute-care and ICU bed hospitalization rate & $\begin{array}{l}\text { Cumulative number of hospital admissions into acute-care } \\
\text { or ICU beds over the cumulative number of documented } \\
\text { PCR laboratory-confirmed infections }\end{array}$ & $\begin{array}{l}\text { Proportion of PCR laboratory-confirmed infections that } \\
\text { progressed to hospital admission into acute-care or ICU } \\
\text { beds }\end{array}$ \\
\hline 2. Crude case severity and criticality rate & $\begin{array}{l}\text { Cumulative number of COVID-19 severe or critical infec- } \\
\text { tions }{ }^{\star} \text { over the cumulative number of documented PCR } \\
\text { laboratory-confirmed infections }\end{array}$ & $\begin{array}{l}\text { Proportion of PCR laboratory-confirmed infections that } \\
\text { progressed to become severe or critical }\end{array}$ \\
\hline 3. Crude case fatality rate & $\begin{array}{l}\text { Cumulative number of COVID-19 deaths over the cumula- } \\
\text { tive number of documented PCR laboratory-confirmed } \\
\text { infections }\end{array}$ & $\begin{array}{l}\text { Proportion of PCR laboratory-confirmed infections that } \\
\text { ended in COVID-19 death }\end{array}$ \\
\hline \multicolumn{3}{|l|}{ Infection rates-model estimation } \\
\hline 1. Infection acute-care bed hospitalization rate & $\begin{array}{l}\text { Cumulative number of hospital admissions into acute-care } \\
\text { beds over the cumulative number of infections, documented } \\
\text { and undocumented }\end{array}$ & $\begin{array}{l}\text { Proportion of infections that progressed to acute-care bed } \\
\text { hospital admission }\end{array}$ \\
\hline 2. Infection ICU bed hospitalization rate & $\begin{array}{l}\text { Cumulative number of hospital admissions into ICU beds } \\
\text { over the cumulative number of infections, documented and } \\
\text { undocumented }\end{array}$ & $\begin{array}{l}\text { Proportion of infections that progressed to ICU bed hospi- } \\
\text { tal admission }\end{array}$ \\
\hline 3. Infection severity rate & $\begin{array}{l}\text { Cumulative number of COVID-19 severe infections }{ }^{\star} \text { over } \\
\text { the cumulative number of infections, documented and } \\
\text { undocumented }\end{array}$ & $\begin{array}{l}\text { Proportion of infections that progressed to become severe } \\
\text { infections }\end{array}$ \\
\hline 4. Infection criticality rate & $\begin{array}{l}\text { Cumulative number of COVID- } 19 \text { critical infections }{ }^{\star} \text { over } \\
\text { the cumulative number of infections, documented and } \\
\text { undocumented }\end{array}$ & $\begin{array}{l}\text { Proportion of infections that progressed to become critical } \\
\text { infections }\end{array}$ \\
\hline 5. Infection fatality rate & $\begin{array}{l}\text { Cumulative number of COVID-19 deaths over the cumula- } \\
\text { tive number of infections, documented and undocumented }\end{array}$ & Proportion of infections that ended in COVID-19 death \\
\hline \multicolumn{3}{|l|}{ Combined infection rates-model estimation } \\
\hline 1. Infection acute-care and ICU bed hospitalization rate & $\begin{array}{l}\text { Cumulative number of admissions into acute-care or ICU } \\
\text { beds over the cumulative number of infections, documented } \\
\text { and undocumented }\end{array}$ & $\begin{array}{l}\text { Proportion of infections that progressed to acute-care or } \\
\text { ICU bed hospital admission }\end{array}$ \\
\hline 2. Infection severity and criticality rates & $\begin{array}{l}\text { Cumulative number of COVID-19 severe or critical } \\
\text { infections }{ }^{\star} \text { over the cumulative number of infections, } \\
\text { documented and undocumented }\end{array}$ & $\begin{array}{l}\text { Proportion of infections that progressed to become severe } \\
\text { or critical infections }\end{array}$ \\
\hline
\end{tabular}

Table 1. Crude case rates and infection rates estimated in this study. ${ }^{\star}$ Per World Health Organization (WHO) infection severity classification ${ }^{11}$.

Figure $1 \mathrm{~B}$ shows the crude case severity and criticality rate versus time. The rate was rather stable, but with a slightly declining trend, and was assessed at 28.0 severe and/or critical cases per 1000 laboratory-confirmed infections on November 22, 2020. As of this date, a total of 4127 severe and 863 critical infections had been registered.

Figure $1 \mathrm{C}$ shows the crude case fatality rate versus time. The rate increased over time, as expected with the weeks-long delay between infection and eventual COVID-19 death, but then stabilized as the epidemic entered its low but stable infection incidence phase (Supplementary Figure S1). The rate was assessed at 13.2 deaths per 10,000 laboratory-confirmed infections on November 22, 2020. As of this date, a total of 235 COVID-19 deaths had been registered.

The model produced robust fits to each dataset. Supplementary Table S3 summarizes the goodness-of-fit. Supplementary Figures S3-S7 show the age-specific posterior distributions of the infection acute-care bed hospitalization rate (Supplementary Figure S3), infection ICU bed hospitalization rate (Supplementary Figure S4), infection severity rate (Supplementary Figure S5), infection criticality rate (Supplementary Figure S6), and infection fatality rate (Supplementary Figure S7). Meanwhile, Supplementary Figures S8-S9 show the overall (total population of all age groups) infection acute-care bed hospitalization rate (Supplementary Figure S8A), infection ICU bed hospitalization rate (Supplementary Figure S8B), infection severity rate (Supplementary Figure S8C), infection criticality rate (Supplementary Figure S8D), and infection fatality rate (Supplementary Figure S9).

Table 2, Figs. 2, and 3A show the estimated mean and 95\% CI of all age-specific infection rate measures. All rates showed very strong age dependence. Measures increased steadily with age, with low values for those $<50$ years of age, but very rapidly growing rates for those $\geq 50$ years of age. The strong age dependence was even more pronounced for infection ICU bed hospitalization rate (Fig. 2B), infection criticality rate (Fig. 2D), and infection fatality rate (Fig. 3A).

The overall (total population of all age groups) infection acute-care bed hospitalization rate was estimated at 13.10 (95\% CI 12.82-13.24) per 1000 infections, infection ICU bed hospitalization rate at 1.60 (95\% CI 1.58-1.61) per 1000 infections, infection severity rate at 3.06 (95\% CI 3.01-3.10) per 1000 infections, infection criticality rate at 0.68 (95\% CI $0.67-0.68)$ per 1000 infections, and infection fatality rate at 1.85 (95\% CI $1.74-1.95)$ per 10,000 infections.

Applying the above estimated age-specific infection fatality rate in Qatar to the age structure of other national populations ${ }^{22}$, the overall infection fatality rate for these countries varied substantially just because of the differences in the demographic structure (Fig. 3B).

Figure $1 \mathrm{D}$ shows the infection acute-care and ICU bed hospitalization rate versus time. The rate increased over time, as expected with the delay between infection and hospital admission, unlike the corresponding crude case 

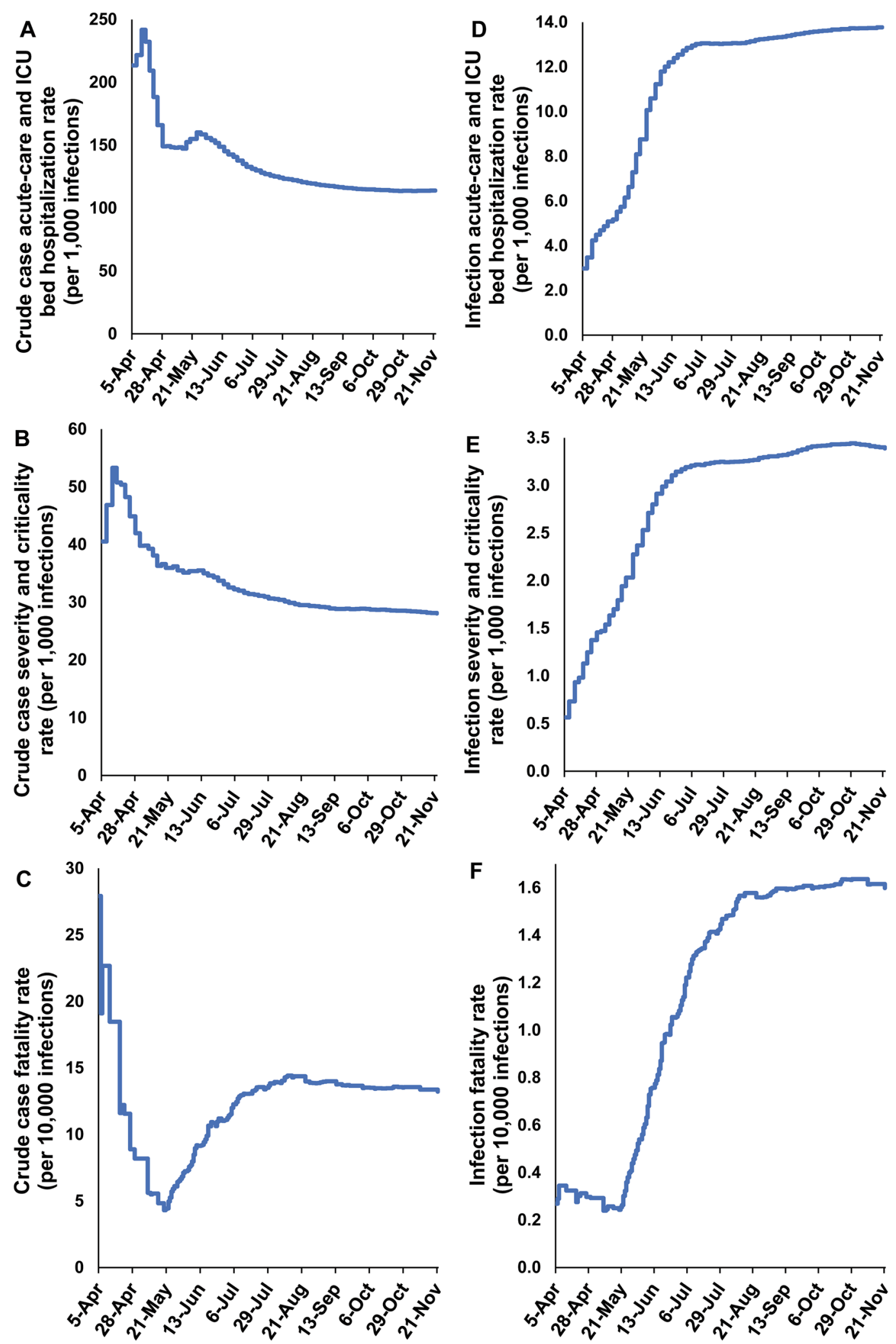

Figure 1. Temporal trend in (A) crude case acute-care and ICU bed hospitalization rate, $(\mathbf{B})$ crude case severity and criticality rate, $(\mathbf{C})$ crude case fatality rate, $(\mathbf{D})$ infection acute-care and ICU bed hospitalization rate, $(\mathbf{E})$ infection severity and criticality rate, and $(\mathbf{F})$ infection fatality rate. Classification of infection severity and criticality was per WHO infection severity classification ${ }^{11}$. 


\begin{tabular}{|l|l|l|l|l|l|}
\hline & $\begin{array}{l}\text { Infection acute-care bed } \\
\text { hospitalization rate (per } \\
\text { 1000 infections) }\end{array}$ & $\begin{array}{l}\text { Infection ICU bed } \\
\text { hospitalization rate (per } \\
\text { 1000 infections) }\end{array}$ & $\begin{array}{l}\text { Infection severity rate (per } \\
\text { 1000 infections) }\end{array}$ & $\begin{array}{l}\text { Infection criticality rate (per } \\
\text { 1000 infections) }\end{array}$ & $\begin{array}{l}\text { Infection fatality rate (per } \\
\mathbf{1 0 , 0 0 0} \text { infections) }\end{array}$ \\
\cline { 2 - 6 } Age group (years) & Mean (95\% CI) & Mean $\mathbf{9 5 \%}$ CI) & Mean (95\% CI) & Mean $\mathbf{( 9 5 \% ~ C I ) ~}$ & Mean (95\% CI) \\
\hline $0-9$ & $8.06(7.85-8.20)$ & $0.44(0.43-0.46)$ & $0.07(0.07-0.08)$ & $0.00(0.00-0.00)$ & $0.00(0.00-0.00)$ \\
\hline $10-19$ & $7.15(6.96-7.27)$ & $0.39(0.38-0.41)$ & $0.13(0.13-0.13)$ & $0.04(0.04-0.04)$ & $0.00(0.00-0.00)$ \\
\hline $20-29$ & $7.19(7.02-7.28)$ & $0.40(0.39-0.41)$ & $0.47(0.46-0.48)$ & $0.08(0.08-0.08)$ & $0.12(0.11-0.13)$ \\
\hline $30-39$ & $10.07(9.88-10.18)$ & $0.71(0.69-0.72)$ & $1.86(1.83-1.89)$ & $0.21(0.21-0.21)$ & $0.09(0.09-0.10)$ \\
\hline $40-49$ & $16.00(15.65-16.17)$ & $1.92(1.90-1.94)$ & $4.05(3.99-4.11)$ & $0.73(0.72-0.73)$ & $0.75(0.70-0.80)$ \\
\hline $50-59$ & $26.35(25.69-26.68)$ & $4.67(4.63-4.70)$ & $8.35(8.22-8.48)$ & $2.10(2.08-2.12)$ & $5.31(4.99-5.65)$ \\
\hline $60-69$ & $60.46(58.82-61.53)$ & $12.65(12.54-12.75)$ & $25.08(24.70-25.48)$ & $7.43(7.37-7.49)$ & $27.68(26.15-29.26)$ \\
\hline $70-79$ & $99.84(97.23-101.65)$ & $38.03(37.72-38.32)$ & $50.16(49.39-50.95)$ & $22.45(22.25-22.62)$ & $116.44(110.43-122.83)$ \\
\hline $80+$ & $36.73(35.76-37.37)$ & $32.94(32.60-33.26)$ & $31.26(30.78-31.76)$ & $23.02(22.82-23.19)$ & $175.76(168.92-183.17)$ \\
\hline Overall population & $13.10(12.82-13.24)$ & $1.60(1.58-1.61)$ & $3.06(3.01-3.10)$ & $0.68(0.67-0.68)$ & $1.85(1.74-1.95)$ \\
\hline
\end{tabular}

Table 2. Estimated mean and $95 \%$ credible interval (CI) of the age-specific infection acute-care and ICU bed hospitalization rates, infection severity and criticality rates, and infection fatality rate. Classification of infection severity and criticality was per WHO infection severity classification ${ }^{11}$.
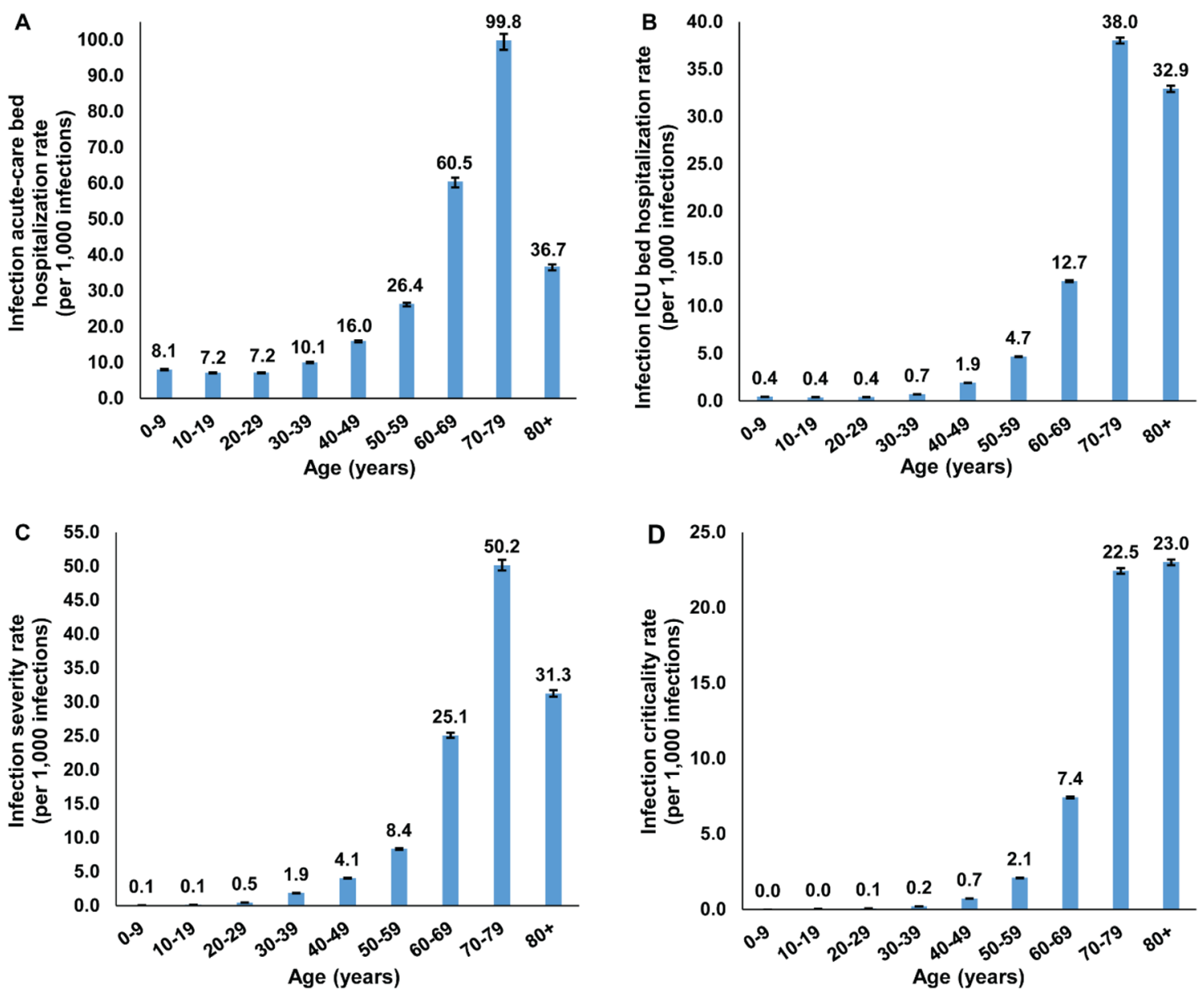

Figure 2. The age-specific (A) infection acute-care bed hospitalization rate, $(\mathbf{B})$ infection ICU bed hospitalization rate, $(\mathbf{C})$ infection severity rate, and (D) infection criticality rate. Classification of infection severity and criticality was per WHO severity classification ${ }^{11}$.

rate which decreased over time as PCR testing was expanded and more and more of the mild and asymptomatic infections were diagnosed. The infection acute-care and ICU bed hospitalization rate then stabilized (Fig. 1D) as the epidemic peaked and started to decline (Supplementary Figure S1). The rate was assessed at 13.8 hospital admissions per 1000 infections on November 22, 2020. A similar pattern was found for the infection severity and 

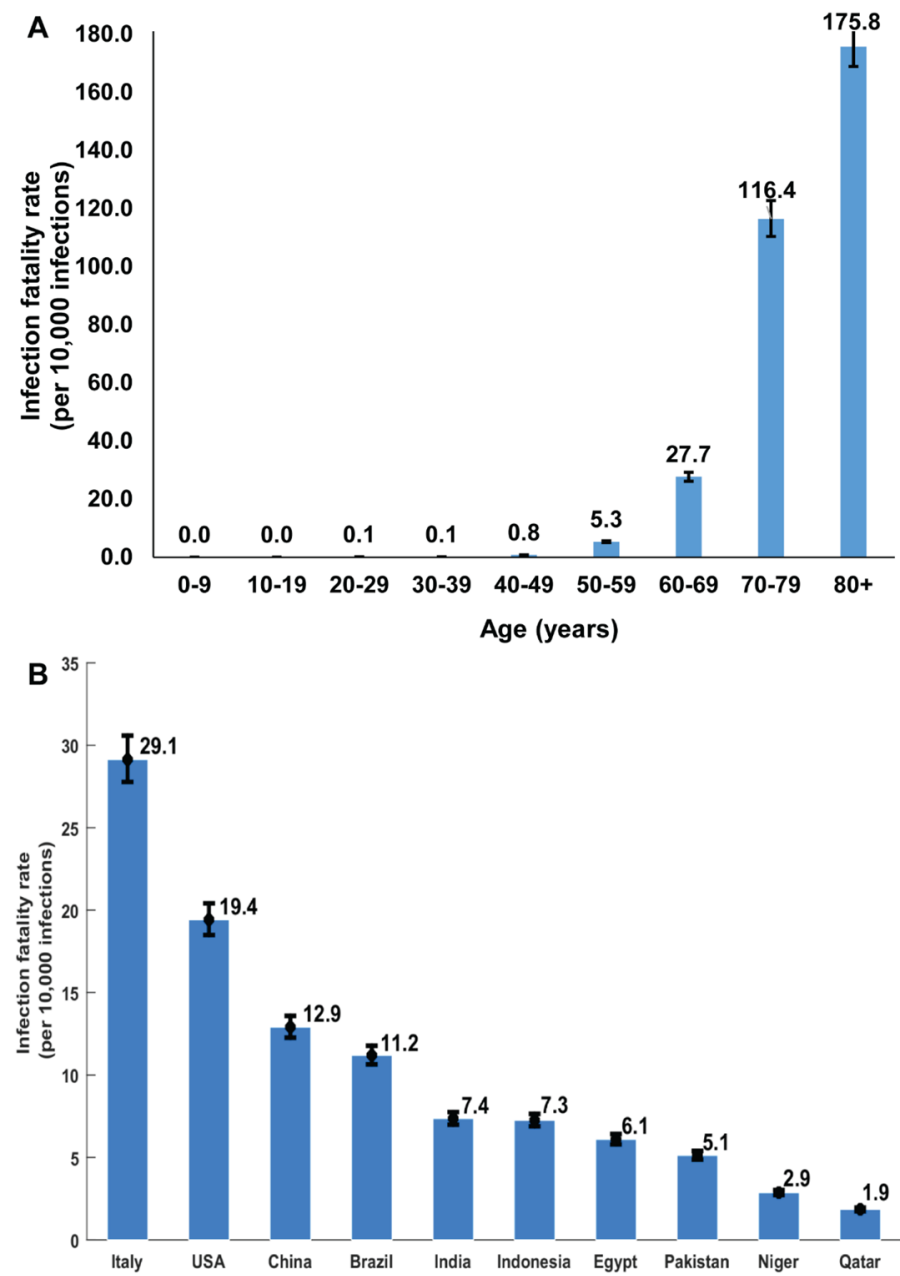

Figure 3. (A) The age-specific infection fatality rate in Qatar. (B) Estimated overall (total population of all age groups) infection fatality rate for several select countries that are characterized by diverse demographic structures. The estimates were generated by applying the Qatar-estimated age-specific infection fatality rate to the age structure of the population in each country. Population size and demographic age-structure of each country were extracted from the United Nations World Population Prospects database ${ }^{22}$.

criticality rate (Fig. 1E) and infection fatality rate (Fig. 1F), which were assessed at 3.4 cases per 1000 infections and 1.6 deaths per 10,000 infections, respectively, on November 22, 2020.

The study generated other relevant estimates. The diagnosis (detection) rate as of November 22, 2020, that is the proportion of infections that were documented out of all infections that were estimated to have occurred, was assessed at $12.1 \%$ (95\% CI $12.0-12.1 \%)$. The mean duration of acute-care hospitalization was estimated at 8.73 (95\% CI 8.62-8.83) days and the mean duration of ICU hospitalization was estimated at 12.30 (95\% CI 12.18-12.41) days.

\section{Discussion}

The striking finding of this study is that SARS-CoV-2 morbidity and mortality demonstrated a very strong age dependence. Infection severity, criticality, and fatality increased very rapidly with age, apart for those $\geq 80$ years of age possibly because of a survival effect. This was particularly the case for infection criticality and fatality which were limited for those $<50$ years of age but increased very rapidly for those $\geq 50$ years of age (Figs. 2, 3). This strong age dependence combined with the lower infection exposure in those $\geq 60$ years of age (Supplementary Figure S10) and the small proportion of the population $\geq 50$ years of age (9\%) and $\geq 60$ years of age ( $2 \%)$, all contributed together to a low morbidity and mortality in Qatar. Out of every 1000 infections, only 3.7 infections were destined to be severe or critical, and out of every 10,000 infections, only 1.9 infections were destined to end in COVID-19 death (Table 2).

Notably, both SARS-CoV-2 morbidity and mortality in Qatar were not much higher than those typically seen in a seasonal influenza epidemic in the United States ${ }^{23,24}$. This fact, however, needs to be interpreted in context. With the young age structure of the population in Qatar, a seasonal influenza epidemic in this country has a much lower severity than that in the United States. Typically, only a handful of influenza-related deaths are reported every year in Qatar ${ }^{25}$. 
Other lines of evidence support these findings. In a survey of ten CMW communities in Qatar, only five severe infections and one critical infection ever occurred in 3233 persons with confirmed infection (antibody and/or PCR positive result), that is an infection severity and criticality rate of 2.5 (95\% CI 1.1-4.9) per 1000 infections $^{8}$. In another nationwide survey of the CMW population, only seven severe infections and one critical infection ever occurred in 1590 persons with antibody and/or PCR positive result, an infection severity and criticality rate of 5.0 (95\% CI 2.2-9.9) per 1000 infections $^{9}$. Both of these estimates are in agreement with the present study estimate of 3.7 (95\% CI 3.7-3.8) per 1000 infections.

These figures, however, are substantially lower than those estimated elsewhere, often using early epidemic data $^{26-34}$. For instance, the infection acute-care and ICU bed hospitalization rate and the infection fatality rate were estimated at 20.4 per 1000 infections and 65.0 per 10,000 infections in the United States, respectively ${ }^{32}$. The fact that the early phases of the epidemic in Europe and the United States heavily affected nursing facilities and care homes of the elderly may have biased many of the early estimates to higher values. It is also possible that our estimates are lower because of the robust accounting of the large pool of undocumented infections in the present study, thanks to the series of serological surveys conducted in Qatar ${ }^{6-9}$. These surveys provided some of the key input data for this modeling study. For instance, the nationwide survey of the CMW population found that only $9.3 \%$ (95\% CI 7.9-11.0\%) of those antibody positive had a prior documented PCR laboratoryconfirmed infection ${ }^{9}$. This is in agreement with the diagnosis (detection) rate estimated here at $12.1 \%$ (95\% CI $12.0-12.1 \%$ ), as well as growing evidence from other countries indicating that only one in every 10 infections was ever diagnosed during the epidemic's first wave $e^{26,35-38}$. The totality of evidence on the Qatar epidemic also indicates that most infections were asymptomatic or minimally mild to be diagnosed ${ }^{5-9,39-41}$. For instance, a national SARS-CoV-2 PCR survey found that $58.5 \%$ of those testing PCR positive reported no symptoms within the preceding two weeks of the survey ${ }^{6}$.

In light of these findings, it is evident that the strong age dependence of SARS-CoV-2 morbidity and mortality is a principal contributor to the low morbidity and mortality seen in Qatar compared to elsewhere. The impact of this strong age dependence is illustrated in Fig. 3B where the age-specific infection fatality rate of Table 2 has been applied to the age structure of other national populations ${ }^{22}$. The infection fatality rate in Italy was found to be tenfold higher than that in Qatar, only because of the population's different age structure. These findings indicate that the infection morbidity and mortality may vary immensely across countries, and will be substantially lower in countries with younger demographic structures, as suggested earlier ${ }^{12}$.

While age appears to be the principal factor, other factors could have also contributed to explaining the low morbidity and mortality in Qatar. Evidence indicates $\mathrm{T}$ cell and antibody reactivity against SARS-CoV-2 in unexposed individuals ${ }^{42-45}$, that probably reflects development of $\mathrm{T}$ cell and antibody immune memory to circulating 'common cold' coronaviruses, which may have in turn led to lower morbidity and mortality ${ }^{42-45}$. The shared-housing dwelling structure in Qatar that contributed to the large SARS-CoV-2 epidemic may have, along with the frequent international travel of Qatar's expatriate population, also contributed to higher levels of exposure to other common cold coronaviruses ${ }^{46,47}$, thereby inducing high levels of broadly cross-reactive $\mathrm{T}$ cell and antibody responses. Such pre-existing immune reactivity ${ }^{42-45}$ may have thus resulted in lower levels of SARS-CoV-2 morbidity and mortality in the population of Qatar.

The resourced healthcare system, which was well below its threshold even at the epidemic peak, may have also contributed to the low observed morbidity and mortality. Emphasis on proactive early hospital admission and treatment, in addition to a cautious approach for SARS-CoV-2 case management, may have limited the number of people who went on to progress to severe or critical disease. This is illustrated in comparing the hospitalization rate in Qatar versus that in the United States, 13.1 versus $20.4^{32}$ per 1000 infections, and the infection fatality rate, 1.9 versus $65.0^{32}$ per 10,000 infections, respectively. Though the infection fatality rate was much lower in Qatar, the hospitalization rate was not too different in the two countries. The cautious approach in Qatar that emphasized hospitalization of COVID-19 patients even when this was not indicated per guidelines may have facilitated provision of medical care promptly when COVID-19 cases started to progress to more severe forms of disease.

Limitations may have affected this study. We estimated rates of infection morbidity and mortality, not accounting for potential differences by sex, comorbidities, or other factors. Model estimations are contingent on the validity and generalizability of input data. Available input data were most complete at the national level, and although there could be subpopulation differences in the highly diverse population of Qatar, these could not be factored in the model given insufficient data at the subpopulation level. Despite these limitations, our model, tailored to the complexity of the epidemic in Qatar, was able to reproduce the observed epidemic trends, and provided profound insights about healthcare needs and infection morbidity and mortality.

In conclusion, SARS-CoV-2 morbidity and mortality demonstrate a strikingly strong age dependence. With its young population structure, both morbidity and mortality were low in Qatar, and not much higher than those typically seen in a seasonal influenza epidemic in the United States ${ }^{23,24}$, but they were substantially lower than COVID-19 morbidity and mortality rates in the United States and elsewhere ${ }^{32-34}$. Out of every 1000 infections, only 3.7 infections were severe or critical, and out of every 10,000 infections, only 1.9 infections were fatal. However, these rates would have been much higher if the population of Qatar had a similar demographic structure to that found in Europe or the United States. These findings suggest that SARS-CoV-2 morbidity and mortality may vary immensely by country or region, and that the pandemic expansion in nations with young populations may lead to considerably milder disease burden than currently believed.

Data availability

All data are available within the manuscript and its supplementary materials. 
Received: 7 February 2021; Accepted: 21 July 2021

Published online: 14 September 2021

\section{References}

1. John Hopkins University Coronavirus resource center. COVID-19 Dashboard by the Center for Systems Science and Engineering (CSSE) at Johns Hopkins University (JHU). https://coronavirus.jhu.edu/map.html. Accessed on July 24, 2020.

2. McKibbin, W. J. \& Fernando, R. The global macroeconomic impacts of COVID-19: seven scenarios. SSRN Electron. J. https://doi. org/10.2139/ssrn.3547729 (2020).

3. Planning and Statistics Authority-State of Qatar. The Simplified Census of Population, Housing \& Establishments. https://www. psa.gov.qa/en/statistics/Statistical\%20Releases/Population/Population/2018/Population_social_1_2018_AE.pdf Accessed on: April 2, 2020. (2019).

4. Planning and Statistics Authority-State of Qatar. Qatar Monthly Statistics. Available from: https://www.psa.gov.qa/en/pages/defau lt.aspx. Accessed on: May 26, 2020. (2020).

5. Al Kuwari, H. M. et al. Epidemiological investigation of the first 5685 cases of SARS-CoV-2 infection in Qatar, 28 February-18 April 2020. BMJ Open 10, e040428. https://doi.org/10.1136/bmjopen-2020-040428 (2020).

6. Abu-Raddad, L. J. et al. Characterizing the Qatar advanced-phase SARS-CoV-2 epidemic. Sci. Rep. 11, 6233. https://doi.org/10. 1038/s41598-021-85428-7 (2021).

7. Coyle, P. V. et al. SARS-CoV-2 seroprevalence in the urban population of Qatar: An analysis of antibody testing on a sample of 112,941 individuals. iScience https://doi.org/10.1016/j.isci.2021.102646 (2021).

8. Jeremijenko, A. et al. Herd immunity against severe acute respiratory syndrome coronavirus 2 infection in 10 communities, Qatar. Emerg. Infect. Dis. 27, 1343-1352. https://doi.org/10.3201/eid2705.204365 (2021).

9. Al-Thani, M. H. et al. SARS-CoV-2 infection is at herd immunity in the majority segment of the population of Qatar. Open Forum Infect. Dis. https://doi.org/10.1093/ofid/ofab221 (2021).

10. Hamad Medical Corporation. National SARS-CoV-2 PCR and antibody testing, infection severity, and hospitalization database. (2020).

11. World Health Organization. Clinical management of COVID-19. Available from: https://www.who.int/publications-detail/clini cal-management-of-covid-19. Accessed on: May 31, 2020. (2020).

12. Ayoub, H. H. et al. Age could be driving variable SARS-CoV-2 epidemic trajectories worldwide. PLoS ONE https://doi.org/10. 1371/journal.pone.0237959 (2020).

13. Ayoub, H. H. et al. Characterizing key attributes of the epidemiology of COVID-19 in China: Model-based estimations. Glob. Epidemiol. https://doi.org/10.1016/j.gloepi.2020.100042 (2020).

14. Makhoul, M. et al. Epidemiological impact of SARS-CoV-2 vaccination: Mathematical modeling analyses. Vaccines https://doi. org/10.3390/vaccines8040668 (2020).

15. Makhoul, M. et al. Analyzing inherent biases in SARS-CoV-2 PCR and serological epidemiologic metrics. medRxiv. doi:https:// doi.org/10.1101/2020.08.30.20184705 (2020).

16. Ayoub, H. H. et al. Mathematical modeling of the SARS-CoV-2 epidemic in Qatar and its impact on the national response to COVID-19. J. Glob. Health 11, 05005 (2021).

17. Makhoul, M., Chemaitelly, H., Ayoub, H. H., Seedat, S. \& Abu-Raddad, L. J. Epidemiological differences in the impact of COVID19 vaccination in the United States and China. Vaccines (Basel) 9, 1. https://doi.org/10.3390/vaccines9030223 (2021).

18. Abu-Raddad, L. J. et al. Characterizing the Qatar advanced-phase SARS-CoV-2 epidemic. medRxiv, 2020.2007.2016.20155317v2 0155312 (non-peer-reviewed preprint) doi:https://doi.org/10.1101/2020.07.16.20155317 (2020)

19. Raftery, A. E. \& Bao, L. Estimating and projecting trends in HIV/AIDS generalized epidemics using incremental mixture importance sampling. Biometrics 66, 1162-1173 (2010).

20. Jonoska Stojkova, B. \& Campbell, D. A. Incremental mixture importance sampling with shotgun optimization. J. Comput. Graph. Stat. 28, 806-820 (2019).

21. MATLAB ${ }^{\circ}$. The language of technical computing. The MathWorks, Inc. (2019).

22. United Nations Department of Economic and Social Affairs Population Dynamics. The 2019 Revision of World Population Prospects. https://population.un.org/wpp/. Accessed on November 1, 2020.

23. Reed, C. et al. Estimating influenza disease burden from population-based surveillance data in the United States. PLoS ONE 10, e0118369. https://doi.org/10.1371/journal.pone.0118369 (2015).

24. Biggerstaff, M. et al. Systematic assessment of multiple routine and near real-time indicators to classify the severity of influenza seasons and pandemics in the United States, 2003-2004 through 2015-2016. Am. J. Epidemiol. 187, 1040-1050. https://doi.org/ 10.1093/aje/kwx334 (2018).

25. Communicable Disease Center. Influenza morbidity and mortality in the State of Qatar. Personal communication on November $22,2020$.

26. Ioannidis, J. P. The infection fatality rate of COVID-19 inferred from seroprevalence data. https://doi.org/10.1101/2020.05.13.20101 253v1. Last accessed July 2, 2020. (2020).

27. Meyerowitz-Katz, G. \& Merone, L. A systematic review and meta-analysis of published research data on COVID-19 infectionfatality rates. Int. J. Infect. Dis. https://doi.org/10.1016/j.ijid.2020.09.1464 (2020).

28. Hauser, A. et al. Estimation of SARS-CoV-2 mortality during the early stages of an epidemic: A modeling study in Hubei, China, and six regions in Europe. PLoS Med. 17, e1003189. https://doi.org/10.1371/journal.pmed.1003189 (2020).

29. Salje, H. et al. Estimating the burden of SARS-CoV-2 in France. Science https://doi.org/10.1126/science.abc3517 (2020).

30. Verity, R. et al. Estimates of the severity of coronavirus disease 2019: A model-based analysis. Lancet 20, 30243-30247. https:// doi.org/10.1016/S1473-3099(20)30243-7 (2020).

31. Brazeau, N. F. et al. Report 34: COVID-19 infection fatality ratio: Estimates from seroprevalence. Imperial Coll. Lond. https://doi. org $/ 10.25561 / 83545(2020)$.

32. Angulo, F. J., Finelli, L. \& Swerdlow, D. L. Estimation of US SARS-CoV-2 infections, symptomatic infections, hospitalizations, and deaths using seroprevalence surveys. JAMA Netw. Open 4, e2033706-e2033706 (2021).

33. Yang, J. et al. Disease burden and clinical severity of the first pandemic wave of COVID-19 in Wuhan, China. Nat. Commun. 11, $1-10(2020)$.

34. Estimated Disease Burden of COVID-19. Center for Disease Control and Prevention. https://www.cdc.gov/coronavirus/2019-ncov/ cases-updates/burden.html. Accessed on June 9, 2021.

35. Anand, S. et al. Prevalence of SARS-CoV-2 antibodies in a large nationwide sample of patients on dialysis in the USA: A crosssectional study. Lancet https://doi.org/10.1016/S0140-6736(20)32009-2 (2020).

36. Havers, F. P. et al. Seroprevalence of antibodies to SARS-CoV-2 in 10 sites in the United States, March 23-May 12, 2020. JAMA Intern. Med. https://doi.org/10.1001/jamainternmed.2020.4130 (2020).

37. Wu, S. L. et al. Substantial underestimation of SARS-CoV-2 infection in the United States. Nat. Commun. 11, 4507. https://doi. org/10.1038/s41467-020-18272-4 (2020).

38. Stringhini, S. et al. Seroprevalence of anti-SARS-CoV-2 IgG antibodies in Geneva, Switzerland (SEROCoV-POP): A populationbased study. Lancet 396, 313-319. https://doi.org/10.1016/S0140-6736(20)31304-0 (2020). 
39. Abu-Raddad, L. J. et al. Assessment of the risk of SARS-CoV-2 reinfection in an intense re-exposure setting. Clin. Infect. Dis. https://doi.org/10.1101/2020.08.24.20179457 (2020).

40. Omrani, A. S. et al. The first consecutive 5000 patients with Coronavirus Disease 2019 from Qatar; A nation-wide cohort study. BMC Infect. Dis. 20, 777. https://doi.org/10.1186/s12879-020-05511-8 (2020).

41. Butt, A. A. et al. Hospital admission rates, length of stay, and in-hospital mortality for common acute care conditions in COVID-19 vs. pre-COVID-19 era. Public Health 189, 6-11. https://doi.org/10.1016/j.puhe.2020.09.010 (2020).

42. Sette, A. \& Crotty, S. Pre-existing immunity to SARS-CoV-2: The knowns and unknowns. Nat. Rev. Immunol. 20, 457-458. https:// doi.org/10.1038/s41577-020-0389-z (2020).

43. Grifoni, A. et al. Targets of T cell responses to SARS-CoV-2 Coronavirus in humans with COVID-19 disease and unexposed individuals. Cell 181, 1489-1501. https://doi.org/10.1016/j.cell.2020.05.015 (2020).

44. Weiskopf, D. et al. Phenotype and kinetics of SARS-CoV-2-specific T cells in COVID-19 patients with acute respiratory distress syndrome. Sci. Immunol. https://doi.org/10.1126/sciimmunol.abd2071 (2020).

45. Ng, K. W. et al. Preexisting and de novo humoral immunity to SARS-CoV-2 in humans. Science https://doi.org/10.1126/science. abe1107 (2020).

46. Al Kahlout, R. A. et al. Comparative serological study for the prevalence of anti-MERS coronavirus antibodies in high- and lowrisk groups in Qatar. J. Immunol. Res. 2019, 1386740. https://doi.org/10.1155/2019/1386740 (2019).

47. Khan, T. et al. Endemic human coronaviruses induce distinct antibody repertoires in adults and children. bioRxiv. doi:https://doi. org/10.1101/2020.06.21.163394 (2020).

\section{Acknowledgements}

We thank Her Excellency Dr. Hanan Al Kuwari, Minister of Public Health, for her vision, guidance, leadership, and support. We also thank Dr. Saad Al Kaabi, Chair of the System Wide Incident Command and Control (SWICC) Committee for the COVID-19 national healthcare response, for his leadership, analytical insights, and for his instrumental role in enacting data information systems that made these studies possible. We further extend our appreciation to the SWICC Committee and the Scientific Reference and Research Taskforce (SRRT) members for their informative input, scientific technical advice, and enriching discussions. We also thank Dr. Mariam Abdulmalik, CEO of the Primary Health Care Corporation and the Chairperson of the Tactical Community Command Group on COVID-19, as well as members of this committee, for providing support to the teams that worked on the field surveillance. We further thank Dr. Nahla Afifi, Director of Qatar Biobank (QBB), Ms. Tasneem Al-Hamad, Ms. Eiman Al-Khayat and the rest of the QBB team for their unwavering support in retrieving and analyzing samples and in compiling and generating databases for COVID-19 infection, as well as Dr. Asmaa Al-Thani, Chairperson of the Qatar Genome Programme Committee and Board Vice Chairperson of QBB, for her leadership of this effort. We also acknowledge the dedicated efforts of the Clinical Coding Team and the COVID-19 Mortality Review Team, both at Hamad Medical Corporation, and the Surveillance Team at the Ministry of Public Health.

\section{Author contributions}

L.J.A. conceived and designed the study and led the mathematical modeling and statistical analyses and writing of the manuscript. S.S. co-constructed and parameterized the mathematical model, conducted the mathematical modeling analyses, and co-wrote the first draft of the manuscript. H.C. contributed to the parameterization of the mathematical model, conducted the statistical analyses, and co-wrote the first draft of the manuscript. H.H.A. co-constructed and parameterized the mathematical model. All authors contributed to data collection and acquisition, database development, discussion and interpretation of the results, and to the writing of the manuscript. All authors have read and approved the final manuscript.

\section{Funding}

The authors are grateful for support provided by the Biomedical Research Program and the Biostatistics, Epidemiology, and Biomathematics Research Core, both at Weill Cornell Medicine-Qatar, the Ministry of Public Health, and Hamad Medical Corporation. The modeling infrastructure was made possible by NPRP grant number 9-040-3-008 from the Qatar National Research Fund (a member of Qatar Foundation). GM acknowledges support by UK Research and Innovation as part of the Global Challenges Research Fund, grant number ES/ P010873/1. The statements made herein are solely the responsibility of the authors. The funders had no role in study design, data collection and analysis, decision to publish, or preparation of the manuscript.

\section{Competing interests}

The authors declare no competing interests.

\section{Additional information}

Supplementary Information The online version contains supplementary material available at https://doi.org/ 10.1038/s41598-021-97606-8.

Correspondence and requests for materials should be addressed to L.J.A.-R.

Reprints and permissions information is available at www.nature.com/reprints.

Publisher's note Springer Nature remains neutral with regard to jurisdictional claims in published maps and institutional affiliations. 
(c) (i) Open Access This article is licensed under a Creative Commons Attribution 4.0 International cc) License, which permits use, sharing, adaptation, distribution and reproduction in any medium or format, as long as you give appropriate credit to the original author(s) and the source, provide a link to the Creative Commons licence, and indicate if changes were made. The images or other third party material in this article are included in the article's Creative Commons licence, unless indicated otherwise in a credit line to the material. If material is not included in the article's Creative Commons licence and your intended use is not permitted by statutory regulation or exceeds the permitted use, you will need to obtain permission directly from the copyright holder. To view a copy of this licence, visit http://creativecommons.org/licenses/by/4.0/.

(C) The Author(s) 2021 